TITRE: LES ALBUMS POUR LA JEUNESSE : LES USAGES DU PÉRITEXTE POUR UN RENOUVELLEMENT DE PRATIQUES DE LITTÉRACIE

Auteur(s): LAurence Allain-Le Forestier, Ph.D., Professeure ESPE Bretagne (VAnNes), CREAD (ESPE BRETAGNE), CELLAM (RENNES2)

PUBLICATION: CRÉATION DE DISPOSITIFS DIDACTIQUES ET ENSEIGNEMENT-APPRENTISSAGE DIVERSIFIÉ EN LITTÉRATIE : VERS UNE VALORISATION DE LA RECHERCHE-DÉVELOPPEMENT ET DE LA RECHERCHEACTION EN ÉDUCATION

PAGES: $6-22$

Directeurs: MARIE-Christine BeAudRy, ISABELle CARIgnAN ET FranÇOIS LAROSE ÉditeuR: LES ÉdITIONS DE L'UNIVERSITÉ DE SHERBROOKE, 2017

ISBN: 978-2-7622-0356-1

URI: HTTP://HDL.HANDLE.NET/11143/9915

DOI: HTTP://DX.DOI.ORG/10.17118/11143/9915 


\section{LES ALBUMS POUR LA JEUNESSE : LES USAGES DU PÉRITEXTE POUR UN RENOUVELLEMENT DE PRATIQUES DE LITTÉRACIE}

Laurence Allain-Le Forestier, Ph.D., professeure

ESPE Bretagne (Vannes)

CREAD (ESPE Bretagne

CELLAM (Rennes2)

Résumé : Les albums pour la jeunesse appartiennent au champ artistique par leurs caractéristiques littéraires et esthétiques. Depuis quelques années, les auteurs/illustrateurs formés à différentes disciplines artistiques s'emparent de l'objet-livre en occupant des espaces tels que couverture, pages de titres, annexes, dédicaces, etc., leur donnant alors une dimension narrative. Dans le cadre d'un renouvèlement de pratiques lectorales à l'école primaire, des élèves de trois classes ${ }^{1}$ d'école élémentaire française ont été soumis à la lecture d'albums particuliers, dans leur usage péritextuel, puis à l'écriture de leur réception visant ainsi à établir un lien entre ces formes étudiées et les effets visés. La problématique qui a gouverné ce travail dans les classes s'articule autour de deux axes : Quel regard les jeunes lecteurs portent-ils sur ces espaces nouvellement investis? Quelles pratiques de lecture/ écriture peuvent s'adapter à ces nouveaux supports? Il semble essentiel de former le jeune lecteur à la découverte et au déchiffrement de ces nouvelles formes.

Mots-clés : album, péritexte, lecture, métalepse, auteur.

1. Notre expérimentation (en cours) porte sur 3 classes de CE2 (2 classes) et CM1 (1 classe). Elle s'intéresse à la réception d'albums dont l'auteur est présent dans le péritexte. Ce qui correspond à des élèves de 8 ans (CE2) et 9 ans (CM1). Les élèves des classes de J-P. Le Pihive (CE2) de l'école Calmette de Vannes (France) et de N. Olivard (CM1) de l'école Anita Conti de Saint-Avé ont participé à cette recherche. 


\section{Problématique}

Comment les jeunes lecteurs adaptent-ils leur lecture à la particularité de l'album ? Dans le cadre d'un renouvèlement de pratiques, lectorales et scripturales, de littéracie, la réception d'œuvres littéraires a été interrogée à travers l'étude de plusieurs albums choisis pour leur usage péritextuel. L'étude porte sur la réception d'albums dont la compréhension ne peut se limiter aux pages internes du récit sans tenir compte du péritexte. Le support album, genre iconotextuel (Montandon, 1990), évolue et de nouvelles formes narratives, jouant avec l'espace péritextuel, apparaissent. Quel regard les jeunes lecteurs portent-ils sur ces espaces nouvellement investis ? Quelles pratiques de lecture/ écriture peuvent s'adapter à ces nouveaux supports? L'usage du péritexte est interrogé dans sa production, c'est-à-dire dans une démarche artistique (du côté des auteurs/illustrateurs) et dans sa réception. Si l'ouvrage présente une complexité narrative due à l'entrelacs de ces formes péritextuelles avec le récit, proprement dit, sa découverte par de jeunes lecteurs est à aménager.

Cette recherche a été menée pendant l'année scolaire 2011-2012 et a porté sur l'étude du péritexte d'albums narratifs, édités entre 1998 et 2013, qui ont fait l'objet de lectures en classe de CE2 et CM1 en France ${ }^{1}$. Le travail a porté, tout d'abord, sur l'étude des pages de garde (dans leur réception essentiellement) puis des dédicaces et des exergues dans leur réception et leur production. Enfin, l'étude a donné lieu à la production d'un écrit, permettant aux élèves d'entrer dans l'univers de l'auteur/ illustrateur. Ces écrits sont de diverses formes : écrits de réception (sur le récit, sur d'autres textes de type «préface »), écrits de création (inventer des dédicaces, des exergues). Dans le cadre de cet article, nous ne présenterons qu'une partie de la recherche portant sur la réception d'œuvres jugées métaleptiques par leur péritexte.

\section{Cadrage}

Lorsqu'un lecteur lit un ouvrage, il rencontre plusieurs éléments qui constituent le livre : le texte écrit par l'auteur (ce pourquoi le lecteur lit le livre) et d'autres textes ou illustrations (qui ne sont pas nécessairement composés par l'auteur). Ces derniers forment le péritexte, que Gérard Genette, dans son ouvrage Seuils, définit par son emplacement « autour du texte, dans l'espace du même volume, comme le titre ou la préface, et parfois inséré dans les interstices du texte, comme les titres de chapitres ou certaines notes » (1987, p. 11).

Ces dernières années, les artistes envahissent les espaces péritextuels tels que couverture, pages de garde, page de titres, préface, postface, exergue, etc. Jusqu'ici, ces espaces étaient considérés comme neutres, car ils ne portaient pas de signification particulière ; or, les créateurs que sont les auteurs/illustrateurs ont investi ces espaces en les « sémantisant ». Si les auteurs s'emparent de formes nouvelles, comment les lecteurs, jeunes de surcroit, reçoivent, perçoivent ces espaces (s'ils les remarquent) ? Les auteurs, par la colonisation du péritexte, engagent une lecture qui nécessite de dépasser le récit lui-même, si l’on considère que le récit débute à la page de titre, et qui s'éloigne de 
la lecture linéaire, lecture d'usage. Quelles relations s'installent entre ces espaces et le récit? Au-delà de l'usage péritextuel des pages de garde et de la dédicace, on peut s'interroger sur la forme et les enjeux de ces espaces et de leur réception par les jeunes lecteurs (Eco, 1979; Jauss, 1978). Enfin, une réflexion sur le renouvèlement des pratiques de littéracie en classe est à mener, en repensant les relations entre le lecteur et le livre.

Dans un souci de diversité, dans le cadre de cet article, nous présentons dans un premier temps deux ouvrages dont l'usage péritextuel mérite l'attention, car il participe à la construction du récit, ce qui illustre ainsi notre objet d'étude. Dans un second temps, nous présentons les réactions d'élèves face à un autre album, Une fois encore! d'Emily Gravett, afin de multiplier les exemples d'usage péritextuel.

\section{Usage péritextuel de l’album : espaces de création}

Seuls deux espaces péritextuels seront analysés: les pages de garde à partir de l'album Le phare des sirènes de Rascal et Régis Lejonc et la dédicace de l'ouvrage Une princesse au palais de Cécile Roumiguière et Carole Chaix.

\section{Les pages de garde}

Dans le domaine de l'imprimerie, les pages de garde ont une existence fonctionnelle. Ce sont par elles que la reliure se fait, et en conséquence, le livre. Elles initient par leur emplacement l'ouverture et la fermeture du livre, dupliquant en quelque sorte l'acte de lecture lui-même. Dans Le phare des sirènes de Rascal et Régis Lejonc, le récit déborde sur les pages de garde et inversement créant une intrication impossible à dénouer sur laquelle le ressort narratif se construit. Ange, le narrateur, revient de la guerre et se remémore sa rencontre avec Swidja, une sirène dont il est tombé amoureux et qu'il a dû quitter précipitamment, car les soldats sont venus le chercher le jour de la mobilisation générale de 1914. II reviendra de la guerre, gueule cassée, meurtri et cherchera à retrouver Swidja. II lui écrira alors une lettre dans laquelle il indique sa position : le phare des sirènes.

La première page de garde, à l'ouverture du livre, donne à lire une première carte : la sirène visible au centre rappelle le titre. 


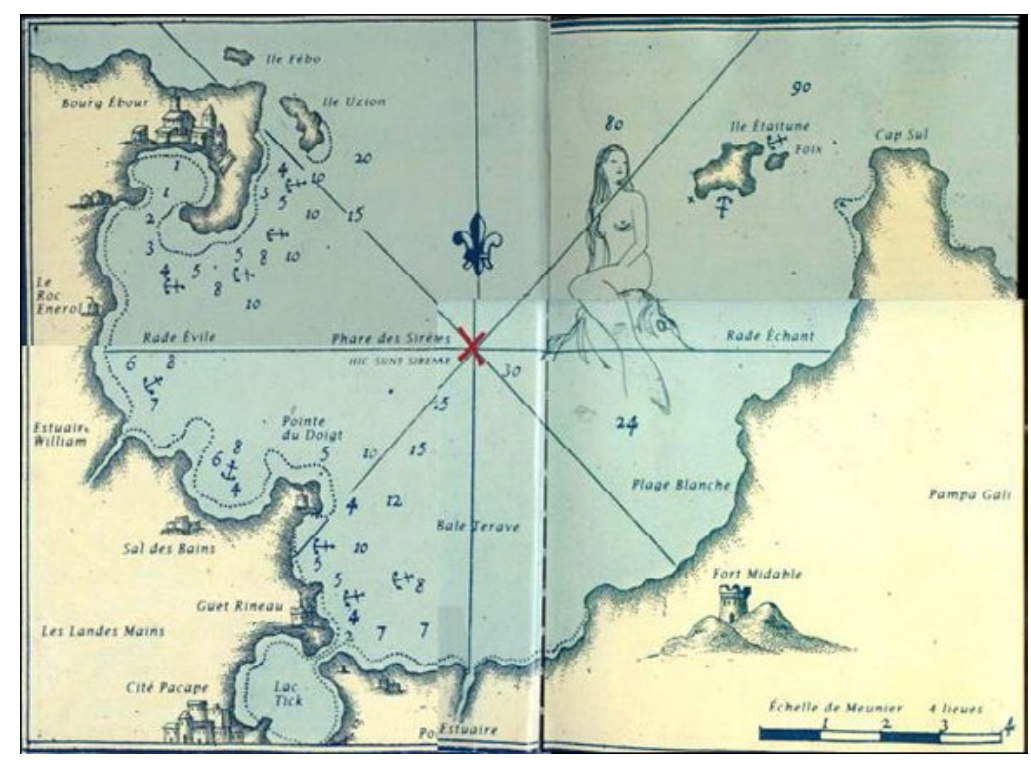

Page de garde initiale de Le phare des sirènes

Après la page de titre, nous découvrons le texte suivant:

Elle est là. A l'endroit exact où bat mon cœur et quelque part sous ces flots bleus.

Je suis là. A l'endroit exact où les cosmographes inscrivaient jadis sur leurs cartes Hic sunt sirenae - Ici sont les sirènes.

$45^{\circ}$ latitude nord.

$35^{\circ}$ longitude ouest.

Phare des sirènes.

Une double page fait suite à ce texte, sur laquelle nous pouvons remarquer un personnage, Ange, le narrateur, écrivant une lettre. Par un effet de zoom, la page de droite nous le montre dessinant, écrivant sur une feuille blanche. La narration est complexe, car elle joue sur les deux codes que sont, d'une part, le texte et, d'autre part, l'illustration ; l'un parlant d'un temps révolu, « II fut un temps où l'on me disait ressembler à mon prénom », et l'autre, l'illustration, signifiant le moment de la narration. Le narrateur dessine, on le comprendra plus tard, une carte. 


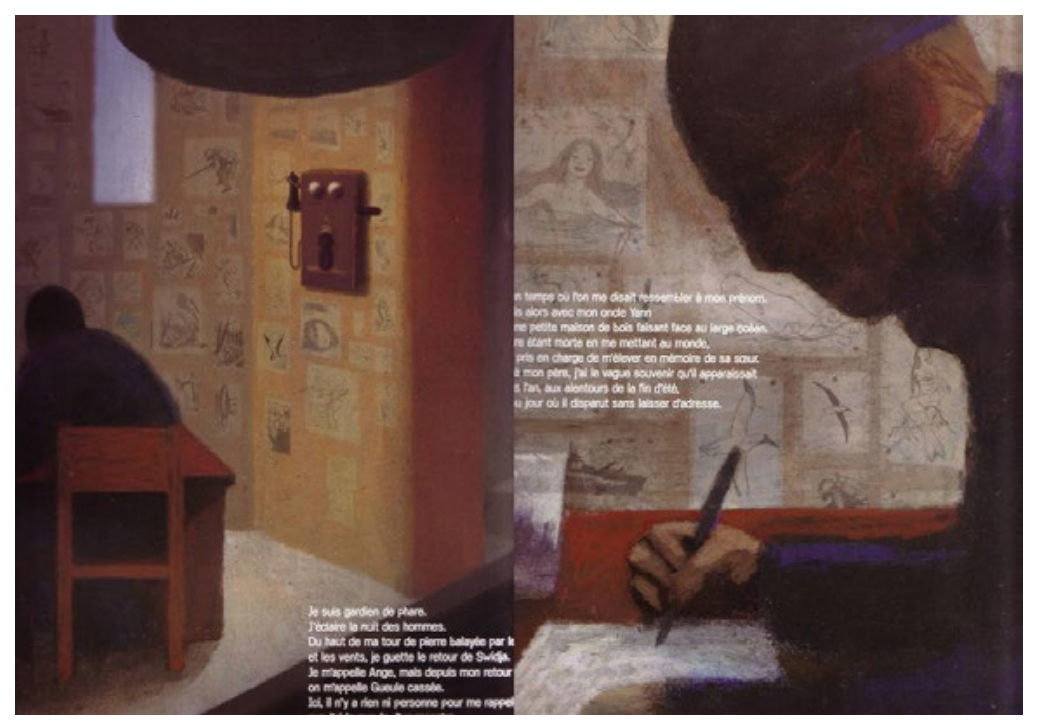

\section{Première double page de l'album Le phare des sirènes}

Le récit est amorcé par le texte et non par l'illustration selon un procédé qui emprunte au fondu sonore du cinéma.

La dernière double page clôt le récit de telle manière que nous retournons au point de départ.

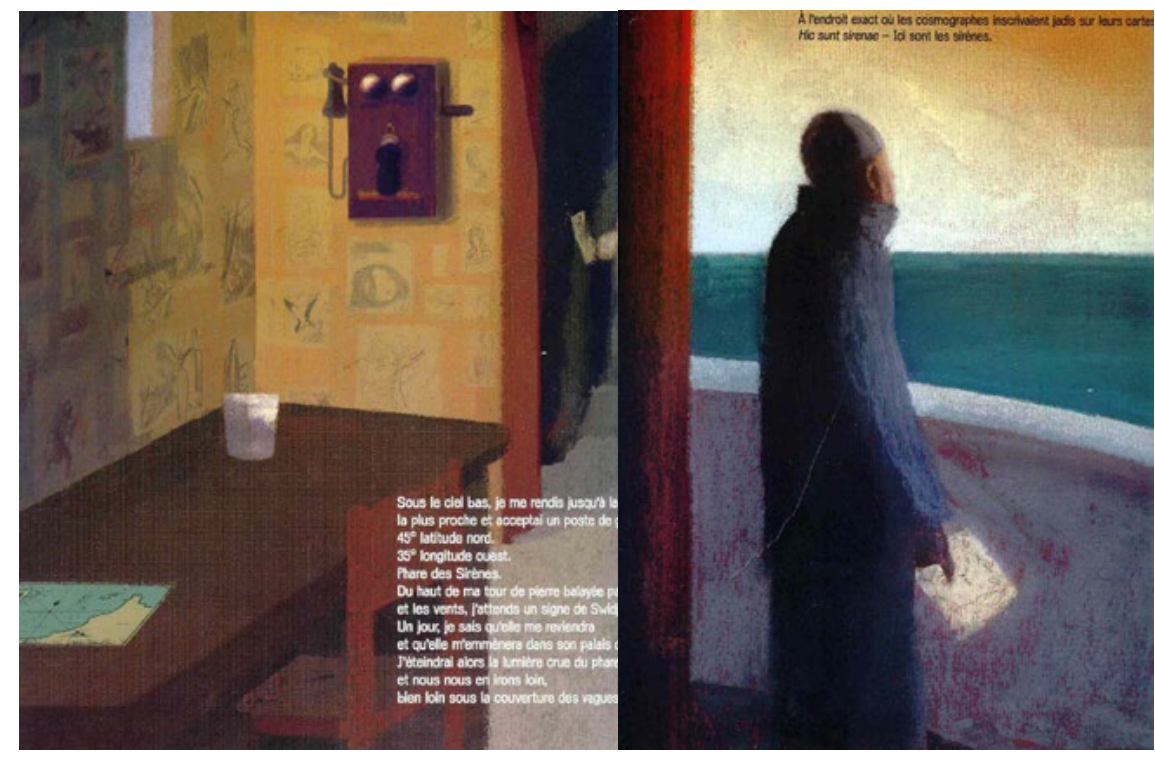

Dernière double page de l'album Le phare des sirènes

Nous remarquons deux cartes: l'une sur la page de gauche qui correspond à la page de garde initiale (image 1) et une deuxième carte, sur la page de droite, qui correspond à la page de garde finale (image 4). 


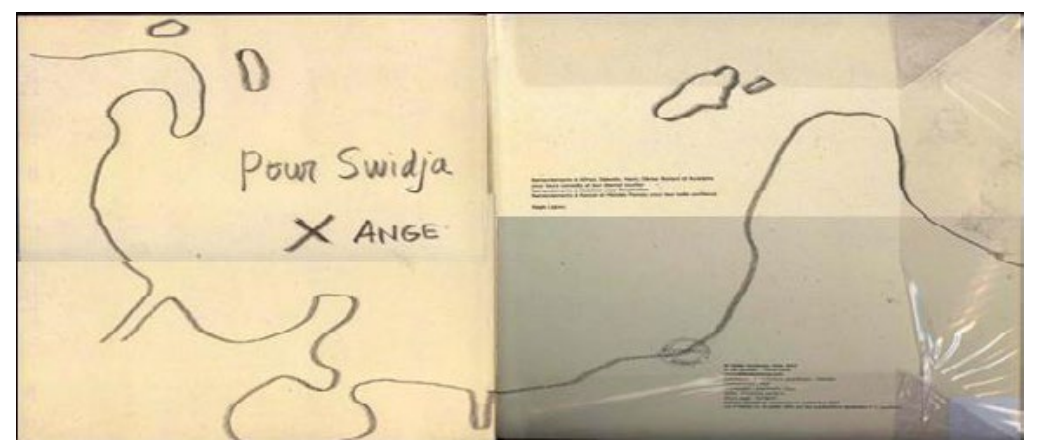

Page de garde finale de l'album Le phare des sirènes

Le récit est triplement enchâssé : enchâssé par la structure narrative elle-même (donc par le texte), enchâssé par l'illustration - les doubles pages qui situent le récit dans le phare et qui permettent de repérer le retour au phare inaugural - et enfin, enchâssé par les pages de garde.

Les éléments péritextuels qui devraient se trouver par nature à la périphérie du texte s'insèrent dans le récit tout en contribuant à sa cohérence. Les pages de garde encadrent le récit et se font écho : la dernière page de garde est épurée par rapport à la première, dépouillée des indications. On y découvre deux inscriptions: une croix qui localise un lieu, le phare où vit Ange, et un destinataire, Swidja, la sirène. Ainsi les deux cartes (l'une en page de garde initiale et l'autre en page de garde finale) désignent-elles le même lieu sans toutefois avoir la même signification : la première ressemble à une carte marine indiquant les dangers du relief et l'autre, beaucoup plus simple, épurée, ne présente que les contours de la côte. Le destinataire de l'une semble être les marins tandis que le destinataire de la dernière est la sirène, qui n’a guère besoin d’indications pour retrouver le lieu.

L'histoire ne se termine pas en refermant le livre : le texte ne dit rien sur la suite de la lettre destinée à Swidja, le texte n’a jamais fait mention de ces cartes, qui sont pourtant bien présentes dans le récit à travers l'illustration et qui jouent un rôle important. Le geste de fermeture du livre s'accompagne naturellement du geste de repositionnement qui met la première de couverture en face du lecteur. S’il est attentif, il établira un lien avec le papier que tient Ange à la fin du livre sur la dernière double page (image 3) et la carte destinée à Swidja, carte agrandie en page de garde finale, carte qu'il dessinait en tout début du livre (dans la première double page, image 2). Alors, la première de couverture prend un autre sens : les morceaux de papier déchiré qui tombent du haut du phare sont les morceaux de la carte destinée à Swidja. Cette première de couverture devient, en définitive, la dernière illustration du récit. 


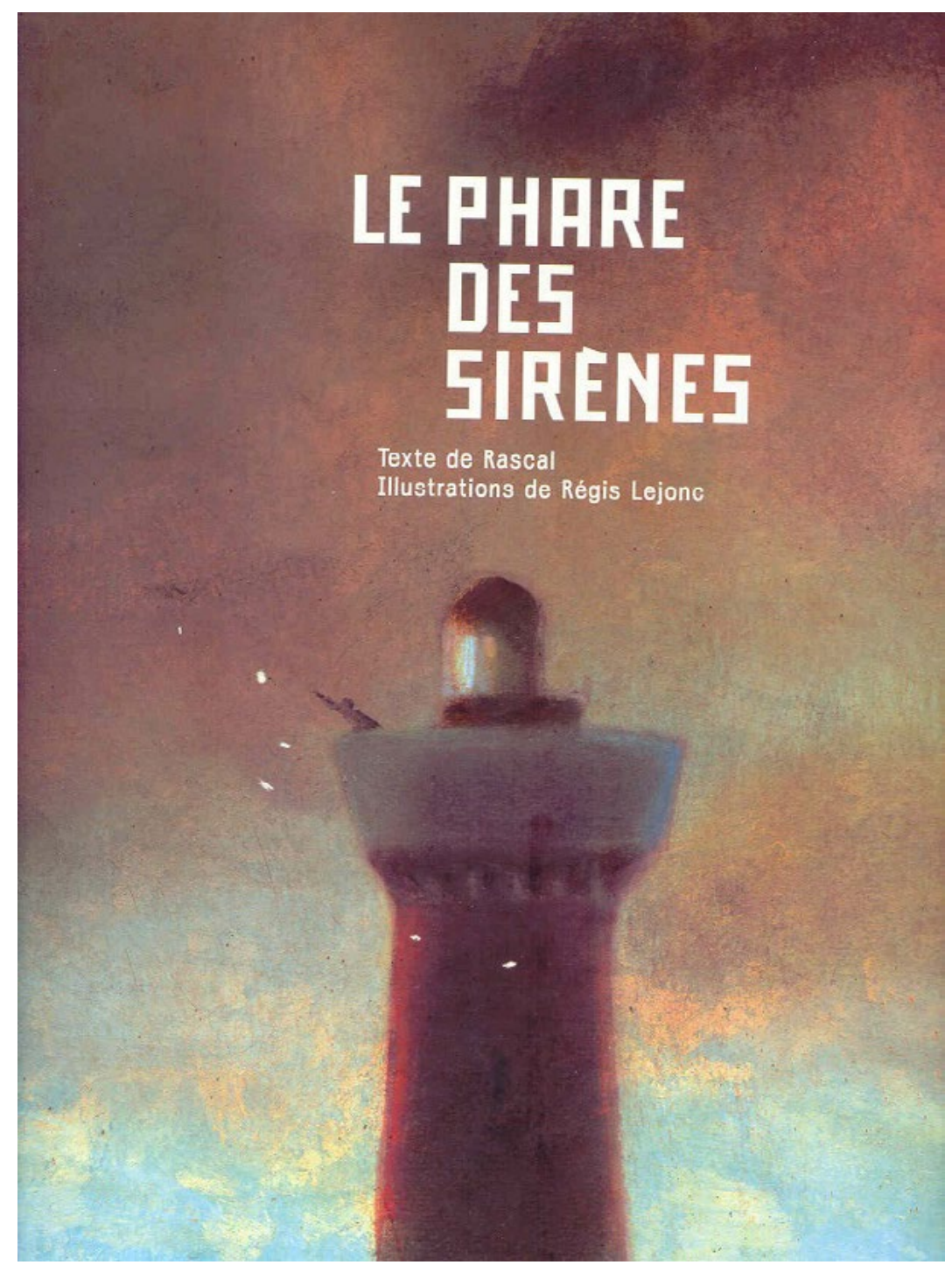

\section{Couverture de l'album Le phare des sirènes}

Le péritexte et le texte sont intriqués, reposant la question du sens de lecture, sens du geste de lecture, et du sens de l'histoire. Le récit se prolonge au-delà de la fermeture de l'album. II oblige à une lecture rétrospective qui cherche à élucider les interrogations nées d'une première lecture insuffisante pour aborder la complexité du récit. Tous les espaces sont brouillés, en interaction permanente, qui traduisent une remarquable collaboration entre Rascal et Régis Lejonc et qui nécessitent un ajustement de la part du lecteur. 


\section{L'espace de dédicace : de la visibilité à la lisibilité}

Les auteurs/illustrateurs s'immiscent dans tous les espaces péritextuels jusque dans les dédicaces, les épigraphes et autres mentions dans lesquels ils se dévoilent, parfois à travers des éléments autobiographiques, parfois à travers des références souvent littéraires. Ce lieu dédicatoire nous semble très intéressant, car il signifie un espace dans lequel le jeune lecteur peut accéder à une forme d'intimité de l'auteur et à sa bibliothèque intérieure (Rouxel, 2004).

L'album Une princesse au palais met en scène une jeune fille dans une brasserie. Elle, une jeune enfant qui devient une jeune fille, attend sa grand-mère, dans la brasserie. C'est l'histoire du passage de l'enfance à la puberté à travers les regards muets des femmes du grand Café, à travers les observations et l'attente de cette jeune fille. Le récit onirique et poétique nous fait comprendre à demi-mots le passage pubertaire de cette petite fille:

ELLE. Elle est là tous les mercredis, ou presque. Elle passe la journée à attendre sa grandmère. Elle se sent bien, elle préfère rester ici qu'être seule à la maison. Elle s'installe à une table, toujours la même, pas loin de la dame antilope. Et elle attend. Ce matin, son ventre est dur, noué. Elle regarde la pendule suspendue à la main de l'ange, et au-dedans d'elle, elle attend. (incipit de l'album)

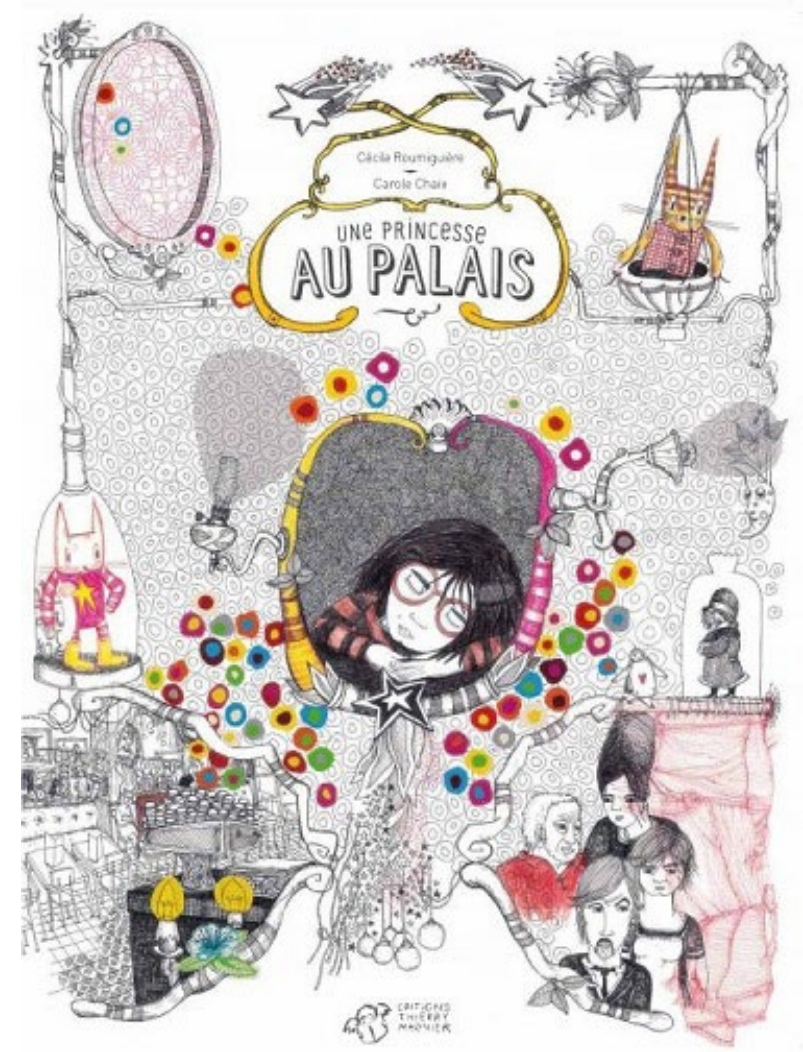

Première de couverture de l'album Une princesse au palais 
L'album, en plus de nous offrir un récit à travers les mots de Cécile Roumiguière et les très belles illustrations de Carole Chaix, nous livre de précieuses informations, des clés interprétatives dans cet espace entre-deux qu'est le péritexte. Le titre avant la lecture peut se comprendre par un jeu de mots créé avec « princesse » et " palais » comme une référence à l'univers des contes où les princesses vivent dans les palais. Ce ne sera qu'après la lecture du récit que le titre pourra s'interpréter grâce à un relevé d'indices dans le récit interne et sur la dernière page mobile, page annexe sur laquelle sont notées plusieurs informations.

Dans la typographie du titre "UNe PRINCeSSe AU PALAIS », nous remarquons l'utilisation d'une seule lettre minuscule, le " e », les autres lettres étant en majuscules. Tout comme Perec refusant d'écrire le «e e, symbole féminin, de la mère dans La disparition (1969), les auteures utilisent le «e » en minuscule, car c'est bien l'histoire d'une petite fille, ELLE, qui deviendra grande. Il nous semble que deux lecteurs se construisent : le premier dans la lecture du récit de cette petite fille devenue grande et le deuxième dans la lecture du péritexte et du récit, l'un éclairant l'autre, l'un rejoignant l'autre.

Le lecteur Modèle (Eco, 1979) est un lecteur savant à l'affût de certains détails qui saura mettre en lien des indices qui ne sont pas visibles dans l'immédiat. Ce lecteur péritextuel est aussi l'adulte, le médiateur, mais aussi le lecteur qu'est devenue Carole Chaix qui illustre l'histoire mise en mots par Cécile Roumiguière ; ce lecteur se double alors de l'image de l'auteur. Sur la dernière page du livre, lieu de rencontre des auteures, page annexe, nous lisons: « Un album librement inspiré du Café du Palais, une brasserie (14, place Myron-Herrick à Reims). Lieu où j'ai passé tant de temps petite, je crois... Merci aux Vogt ». La référence au Café du Palais, que Carole Chaix livre, explique bien des allusions dans le livre. Le lecteur alors se prend à chercher d'autres informations. Il lira ainsi sur cette même page:

L'écume des jours. Boris Vian, 10/18 éd., 1978, page 9

Night and day, chanson de Cole Porter, 1932

Alice's Adventures Wonderland, Lewis Caroll, 1865

ANAIIS NIN, MARGUERITE DURAS, NANCY HUSTON, ANNIE ERNAUX, et toutes les femmes qui se sont emparées des mots.

Le lecteur Modèle est un lecteur savant, sans doute féminin, qui reconnaîtra les références des auteures, reconnues comme auteures féministes, qui ont traité de la vie des femmes, de leur intériorité et de leur condition. Par ailleurs, les quatre auteures citées ont écrit leur autobiographie ou autofiction. Le péritexte nous permet ainsi de construire un faisceau de liens qui, mis bout à bout en relation avec le récit, autorisent une interprétation: Cécile Roumiguière raconte le récit intime de Carole Chaix, autrement dit, une autofiction que l'on découvre dans des lieux réels, tel le décor que l'on peut reconnaitre. À la liste des auteures reconnues comme littéraires et féministes, nous pouvons ajouter les noms de Cécile Roumiguière et Carole Chaix qui traitent, dans une forme d'autofiction, 
d'une condition féminine, d'une transformation féminine importante. Ce lecteur (devrions-nous dire, cette lectrice ?) est ainsi un lecteur lettré, savant, qui possède les capacités cognitives lui permettant d'inférer et d'émettre des suppositions. Il est probable qu'il se distingue du lecteur du récit qui sera moins lettré, mais tout aussi capable d'opérations cognitives.

Une image d'auteur, qui dans les pages annexes fait référence à des réalités, se dégage plus nettement dans cet espace dédicatoire que l'on peut alors considérer comme lieu de rencontre à découvert de l'auteur avec son lecteur : celui-là lira dans les marges du livre jusqu'à la dernière page de garde où mêmes les mentions légales nous livrent un peu de plaisir et d'humour : "Achevé d'imprimer à l'heure du café....»

L'album lu, le péritexte découvert, il reste une troisième lecture à effectuer, celle de la relecture à la lumière de ces informations. Quelles images des quatre auteures citées peut-on retrouver dans le texte? Quelles allusions ou références à Alice peut-on (re)découvrir? Quelles références à la réelle brasserie, le Café du Palais? Quelles allusions à la vie de Carole Chaix? Le péritexte délivre des informations qui sont autant de clés de relecture et d'interprétation textuelle.

\section{Réceptions observées : quel regard les jeunes lecteurs portent sur ces espaces péritextuels ?}

Plusieurs de ces ouvrages ont été soumis à de jeunes lecteurs de CE2 (qui correspond à la tranche d'âge de 8-9 ans). L'album Une fois encore! d'Emily Gravett (2011) a fait l'objet d'un travail dans cette classe, car il a la particularité d'utiliser tous les espaces péritextuels et surtout d'utiliser une jaquette, ce qui est assez peu usité dans les albums. Cette lecture a été réalisée par petits groupes de dix élèves qui ont été guidés par le questionnement de l'enseignant. Tous les élèves de la classe ont rencontré cet ouvrage, soit par l'intermédiaire de l'enseignant ou en lecture autonome. Puis un retour en groupe classe entière a permis, à l'aide du Tableau Blanc Interactif, de revenir sur les remarques des élèves, remarques de nature différente selon qu'ils avaient lu en autonomie ou pas.

L'auteure fait feu de tout bois, avec un grand talent, utilisant le péritexte dans sa totalité : la jaquette, la couverture du livre, les pages de garde et autres pages de titre et faux-titre. Les élèves ont déjà suivi une première séance sur l'étude du péritexte et commencent à utiliser les termes conventionnels tels que première de couverture, pages de garde, etc. 


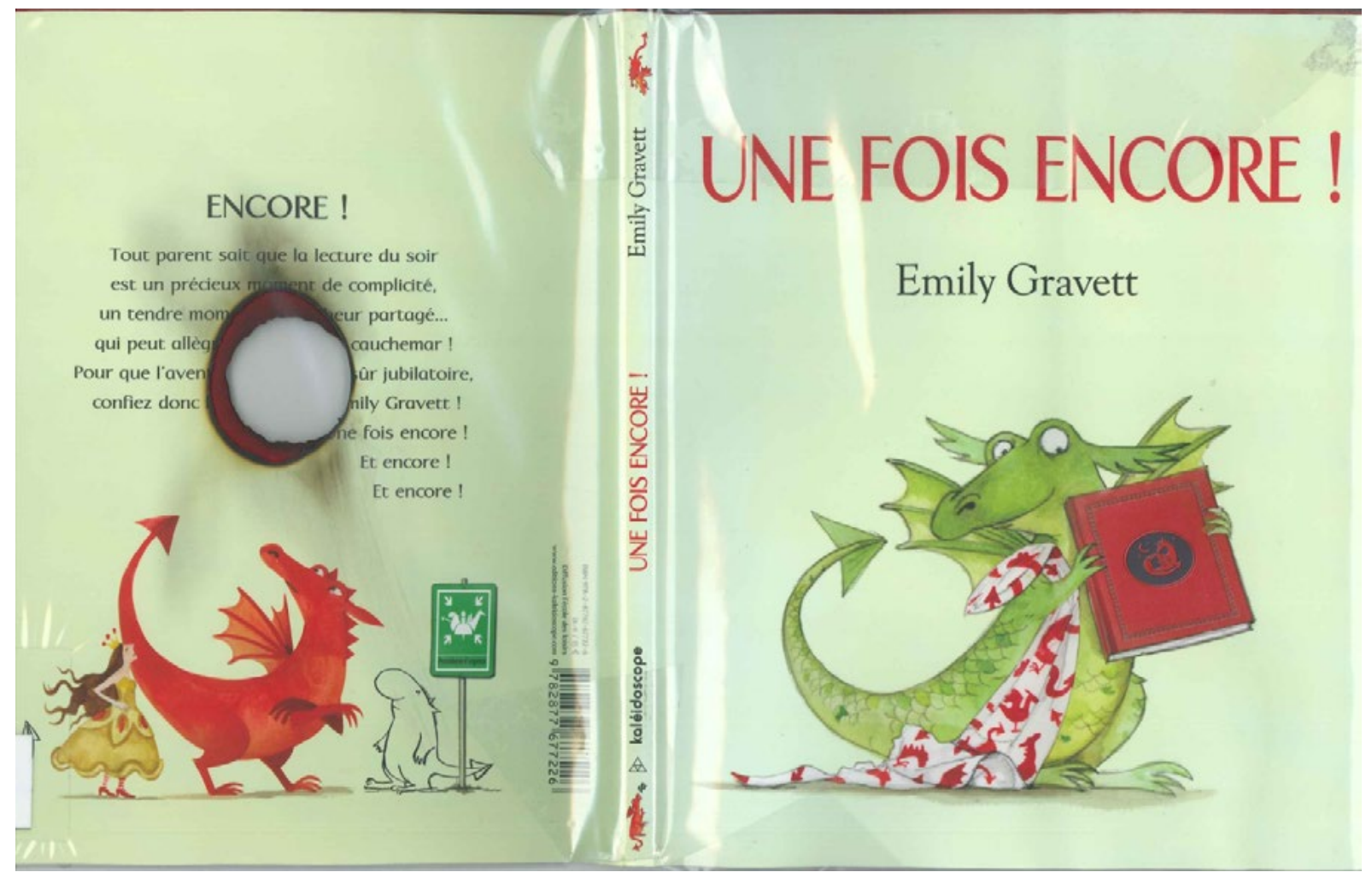

Couverture de l'album Une fois encore !, E. Gravett

Un jeune dragon demande à son père (mère ?) dragon de lui lire un livre dans lequel on découvre un dragon nommé Cédric. Une fois la lecture achevée, le jeune dragon, qui écoute l'histoire racontée par son père, demande une deuxième lecture : «Une fois encore ! »; le père s'exécute mais commence à changer les mots du texte afin d'écourter le temps de lecture. Le jeune dragon ne l'entend pas ainsi et réclame : «Une fois encore! »... et cela, plusieurs fois, laissant notre héros insatisfait, car le père finit par s'endormir. Il n'en faudra pas plus pour que le jeune dragon s'énerve : ainsi se met-il à cracher du feu sur le livre, ce qui a pour effet de le trouer. Le trou est matérialisé : le livre que nous tenons, qui ressemble de plus en plus à celui du petit dragon, présente également un trou dans la jaquette. Le feu craché par le jeune dragon a dépassé les frontières du récit puisqu'il a réussi à bruler la page que nous avons sous la main et sous les yeux. 
L’ouvrage est découvert en petit groupe : la lecture commence par les pages de garde...

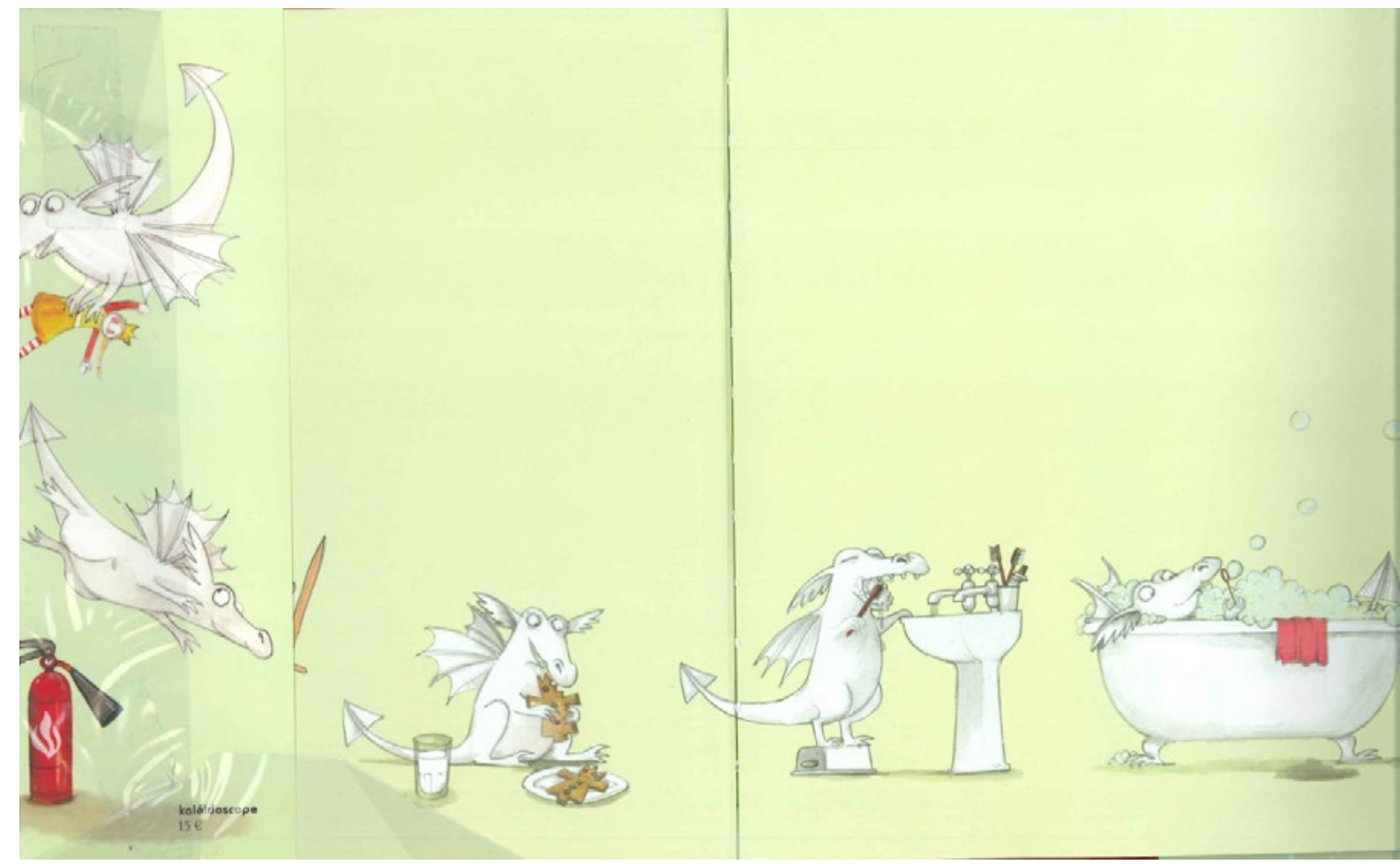

Page de garde initiale de l'album Une fois encore !, E. Gravett

... puis les pages de faux-titre et de titre, redoublées, qui semblent identiques. En effet, ces deux pages diffèrent d'un point d'exclamation et de quelques bulles de savon... Les élèves font le lien entre la première page de garde et la page de faux-titre par les illustrations (image 8 où le dragon prend son bain), le redoublement de la page de titre est tout de suite compris:

Maelys 10 : Là, y a encore « une fois » (découverte de la page de titre). Y a encore pareil que la page d'avant, c'est exactement pareil.

Lola 13 : Ah oui, j’ai compris, j'ai compris.

Lola 15 : «Encore une fois » ça veut dire que ça va être encore une fois ça, encore une fois ça, encore une fois.

Sans être dupes, ils comprennent déjà que nous sommes dans un leurre:

Maelys 20 : Mais ça va pas faire toujours parce que sinon le livre, ça va pas faire d'histoire.

Ils élaborent une signification tout en étant conscients que l'auteur s'amuse avec son lecteur. La fin de l'histoire nous amène à relire à nouveau les pages de garde, initiales et finales, et à les comparer. Certes, ce n'est pas un geste naturel, mais il contribue à développer des compétences lectorales. Les élèves font des liens entre la page de garde initiale et le récit: 
E 43 : Bon là il joue, il se brosse les dents, il va prendre son bain, on voit les bulles.... Il était sorti du bain et après quand il est sorti du bain, il veut...

Groupe 45 : Avant d'aller au lit, avant de lire son livre.

Puis, nous interrogeons cette fois la page de garde finale:

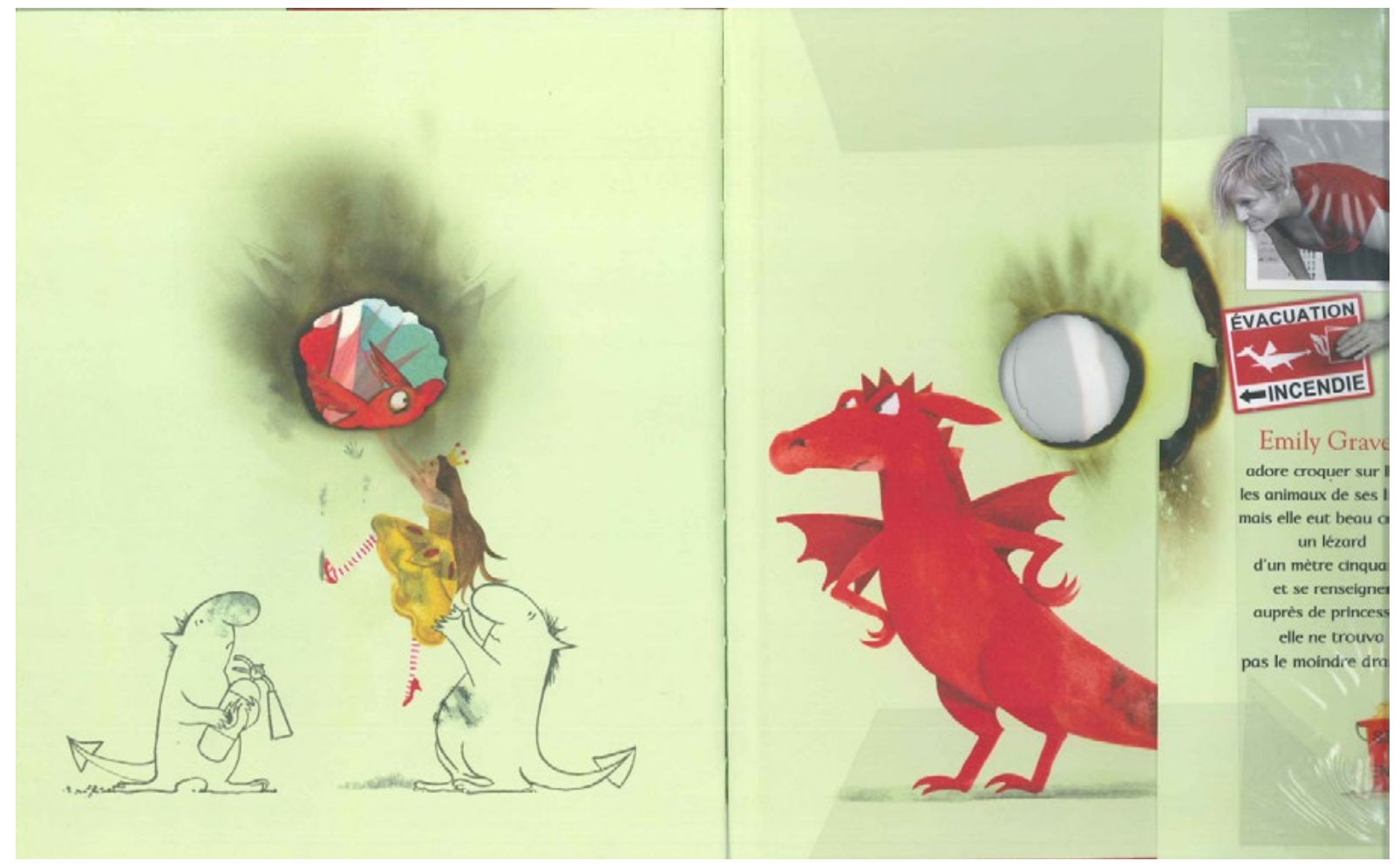

Page de garde finale de l'album Une fois encore !, E. Gravett

M 47 : Est-ce que ça nous donne des explications sur ce qu'on vient de lire?

E 48: Oui parce que là, le dragon il a craché du feu donc ça a déchiré le livre... et là aussi ça a déchiré le livre, en fait il est pas content... Les personnages du livre d'ici (l'élève tourne la page et montre avec son doigt) et ben ils sont revenus ici.

En définitive, tout fait sens dans le livre de Gravett ; les élèves abordent quelque chose de très complexe en littérature, soit la notion de mise en abyme. À cela s'ajoutent toutes les transgressions que l'auteure réalise, que nous appelons des métalepses.

En s'intéressant à d'autres espaces que celui investi entre la page de titre et la dernière page mobile, les lecteurs construisent des significations qui éclairent, parfois trompent leur compréhension première. La lecture ne peut être linéaire, elle appelle, dans cet ouvrage particulièrement, des relectures, des allers-retours entre les pages de garde, la jaquette, les pages de titre, etc. Emily Gravett 
s'amuse, brouillant les repères matériels du péritexte. La mise en abyme trouve son apogée dans cet album, par le texte et l'image, par la matérialité de l'objet-livre, et par la couverture et son jeu de dissimulation. Assurément, cet album transgresse toutes les frontières, par le jeu des métalepses d'ordre ontologique et rhétorique. Les élèves ne s'y trompent pas : la jaquette dissimule quelque chose que nous, lecteurs, retrouvons à l'intérieur du livre que nous lisons. En effet, le livre recouvert (devrions-nous dire dissimulé ?) par la jaquette ressemble étrangement au livre que le jeune dragon, Cédric, tient et que l'adulte tente de lui lire.

E 75 : Ben ça c'est la même couverture que le livre que sa maman lit au dragon.

Maelys 77: On sait parce que là y a un dragon déjà, là y a la lune et dans l'histoire on sait qu'il dort jamais, et là y a la lune et là y a un livre comme dans l'histoire, y a des étoiles et y a un dragon rouge.

Nettie 79 : Je pense que ce dragon là c'est celui de l'histoire.

E 80 : Moi je suis pas du tout d'accord avec Maelys et Nettie, parce que si t'as pas lu l'histoire, Ok tu sais qu'il y a un dragon mais si tu ouvres pas la page et que tu regardes bien et que tu lis pas l'histoire tu vas pas savoir qu'il y a un livre dans l'histoire.

Le livre met ainsi en scène un récit dès la première de couverture (bien que dans ce cas la notion de couverture soit également à interroger) jusqu'à la dernière. Tout fait sens : l'objet-livre lui-même, les rabats de la jaquette, le récit. Les élèves s'emparent de tous ces espaces dès que la situation pédagogique les incite à reconstituer le sens. Les relations lecteur-auteur-livre sont ainsi à reconsidérer.

\section{Discussion}

\section{Peut-on renouveler les pratiques de lecture? Pour quelles compétences?}

Les élèves sont sensibles à ces nouveaux espaces repérés lors de la découverte des albums. Il convient, nous semble-t-il, de poser la question du renouvèlement de pratiques lectorales adaptées à ces supports. Et surtout, il convient de s'interroger sur le développement de compétences qui en découlent. Si la littéracie est définie comme la «capacité d'une personne de comprendre et d'utiliser des imprimés et des écrits nécessaires [souvent à l'écran] pour fonctionner dans la vie de tous les jours, à la maison, au travail et dans la collectivité, pour atteindre ses objectifs, parfaire ses connaissances et réaliser son potentiel » (OCDE et Statistique Canada, 1995), il semble nécessaire d'accommoder cette faculté de lecture à tous types d'écrits, en particulier ceux qui s'adressent à la jeune génération en devenir. 
Les albums rencontrés en classe présentent un rapport espace-temps différent. La temporalité, propre à la structure narrative, s'associe à la mise en espace, propre aux arts visuels dans un jeu d'illusions. La lecture magistrale du texte est alors insuffisante à rendre compte de la complexité de l'œuvre et insuffisante à développer un regard esthétique. L'approche magistrale de ces œuvres est donc à repenser.

Le jeu métaleptique, car nous définissons dans ces exemples le péritexte comme une « métalepse péritextuelle » du fait de son débordement des seuils, est complexe et s'appréhende dans l'organisation de l'album, dans sa découpe de pages et son architecture globale. Les élèves sont capables, dans des lectures complexes telles que celles que nous venons d'évoquer, de repérer les jeux que l'auteur/illustrateur installe, comprenant à la fois l'intention esthétique et s'amusant de ces effets. Une double compétence alors se construit; d'une part, celle du lecteur critique, distancié, lecteur savant qui s'inscrit dans les pages du livre et, d'autre part, celle du lecteur naïf qui se prend dans le jeu de l'histoire. Les deux lecteurs sont en action, l'un dépassant parfois l'autre, jamais à leur détriment mais davantage dans une sorte de collaboration.

Ainsi, quelques pistes de réflexion nous semblent pertinentes à ouvrir, permettant de renouveler les pratiques de lecture adaptées aux albums dont le péritexte fait sens. La relecture devient une lecture indispensable à la construction du sens, en s'appuyant en particulier sur le péritexte. Cette relecture n'est pas une deuxième lecture, elle diffère de la première par son intention et sa compétence. En outre, le développement d'une pensée critique peut s'exercer à la fois par la réflexion suscitée par les citations en exergue, par les éléments dédicatoires qui traduisent un rapport au monde que l'auteur exprime et dont le jeune lecteur peut s'emparer pour peu qu'il y ait été invité. Enfin, la lecture de ces albums et leur étude devient une forme d'engagement à l'écriture créative en proposant aux élèves d'inventer des seuils (dédicace, exergue, pages de garde, etc.) après la lecture d'albums particuliers, par le biais du cahier de littérature. La pratique d'atelier d'écriture pourrait également trouver des idées à emprunter à ces albums.

\section{Conclusion}

En conclusion, les artistes donnent de plus en plus souvent une signification à l'espace péritextuel. Les artistes considèrent l'album comme un tout, un objet artistique, et ce, dans tous ses espaces. Ils invitent implicitement les lecteurs à les investir. La lecture naturelle d'un album reste la lecture linéaire, dans l'ordre des pages certes, mais si l'on considère que l'album appartient à un champ artistique, il peut, tout comme une lecture d'œuvre picturale, bénéficier d'un traitement particulier qui diffère dès lors d'une lecture plus traditionnelle. La lecture de certains éléments péritextuels après la lecture intégrale de l'album pourrait, en partie, et ce, pour certains ouvrages, permettre de construire une interprétation. La lecture s'adapte à son support ; l'album de jeunesse récent et moderne, voire postmoderne, offre un autre rapport au texte, complètement nouveau. Cette nouveauté est à interroger par le renouvèlement de pratiques scolaires. 


\section{Références}

Eco, U. (1979). Lector in fabula. Le rôle du lecteur ou la Coopération interprétative dans les textes narratifs. Paris, France : Grasset.

Genette, G. (1987). Seuils. Paris, France : Seuil.

Gravett, E. (2011). Une fois encore! Paris, France : Kaléidoscope.

Jauss, H. R. (1978). Pour une esthétique de la réception. Paris, France : Gallimard.

Montandon, A. (dir.) (1990). Iconotextes. Paris, France : Ophrys, Centre de Recherche en Communication et Didactique.

OCDE et Statistique Canada (1995). Littératie, économie et société : résultats de la première Enquête internationale sur l'alphabétisation des adultes, Statistique Canada, n 89-545F au catalogue. Paris : OCDE; Ottawa : Ministère de l'Industrie du Canada.

Perec, G. (1969). La disparition. Paris, France : Denoël.

Rascal et Lejonc, R. (2007). Le phare des sirènes. Paris, France : Didier Jeunesse.

Roumiguière, C. et Chaix, C. (2012). Une princesse au palais. Paris, France : Thierry Magnier.

Rouxel, A. (2004). Autobiographie de lecteur et identité littéraire. Dans Rouxel, A. et Langlade, G. (dir.), Le sujet lecteur. Lecture subjective et enseignement de la littérature (p. 137-152). Rennes, France: Presses universitaires de Rennes. 